\title{
Epidemiological Characteristics of Colorectal
}

\section{Cancer and Polyp}

\author{
Masood Ghane ${ }^{1}$, Mitra Ameli ${ }^{2}$ and Sahar Mehrabani Khasraghi ${ }^{1{ }^{*}}$ \\ ${ }^{1}$ Department of Microbiology, Tonekabon Branch, Islamic Azad University \\ Tonekabon, Mazandaran, Iran \\ ${ }^{2}$ Department of Medicine, Tonekabon Branch, Islamic Azad University \\ Tonekabon, Mazandaran, Iran \\ ${ }^{*}$ Corresponding author
}

\begin{abstract}
Copyright (C) 2014 Masood Ghane, Mitra Ameli and Sahar Mehrabani Khasraghi. This is an open access article distributed under the Creative Commons Attribution License, which permits unrestricted use, distribution, and reproduction in any medium, provided the original work is properly cited.
\end{abstract}

\begin{abstract}
Colorectal cancer is the third leading cause of cancer related deaths in the world, with one million new cases detected each year. The purpose of this study was to evaluate tumor characteristics, polyp and some other demographic indicators in patients were admitted to the Endoscopy clinic of Toos and Firoozgar hospital in Tehran, Iran and compare it with other countries. In this study, 35 patients were admitted to the Endoscopy clinic between January 2013 and May 2013. Demographic data, pathologic and colonoscopy findings was performed with SPSS software and chi square test. From 35 patients, 15 patients $(42.8 \%)$ with colorectal tumors and 20 patients $(57.1 \%)$ were diagnosed with colorectal polyps. In patients with colorectal tumors, adenocarcinoma in $100 \%$ of cases and the most common anatomic site of involvement was rectum (33.3\%). Type of colorectal polyps, hyperplastic in $75 \%$ of cases, adenomatus tubular in $15 \%$ and adenomatus villus in $10 \%$ and the most common anatomic site of involvement was sigmoid (25\%). Statistical analysis showed no significant association between anatomic location and incidence colorectal cancer and polyps $(\mathrm{P}=0.169)$. Cancer screening
\end{abstract}


program in patients with a family history of colorectal especially in patients with rectum and sigmoid sites cancer is essential, why hide the symptoms of this type of cancer its later shows.

Keywords: Colorectal cancer, Polyp, Epidemiology

\section{Introduction}

Colorectal cancer is the most common gastrointestinal cancer and the leading of cancer deaths in the United States of America (Burt, 2000). That about 10\% of deaths due to cancer is accounted for. Regarding the gender factor of colorectal cancer among women, after lung and breast cancer and in men after lung and prostate is the third rank of incidence. According to the word health organization (WHO), each year 875000 new cases of the disease are recorded (Janaut \& Kollarova, 2001). The disease usually occurs in older people, so that more than $90 \%$ of patients are diagnosed after age 55 years. The incidence of colorectal cancer varies around the world, in America, Western Europe, Australia and Japan has the largest rate and in African and Asian countries has the lowest rate (Parkin et al., 2005). The majority of colorectal cancers (regardless of the etiology) of adenoma polyps originate. Adenomatous polyps in the colon approximately $30 \%$ of middle-aged and $50 \%$ of older adults seen, but less than $1 \%$ are malignant polyps. Adenomatous polyps may be pedunculated or sessile, cancer is more common in sessile types. Adenomatous polyps could be tubular histology, villus and tubular-villus. The probability of malignancy in adenomatous villuses are 3 equivalent adenomatous tubular. Over the years, numerous risk factors associated with colorectal cancer have been identified, including feeding practices, obesity, lack of exercise, eating alcohol and smoke, crohn's disease, ulcerative colitis, adenomatous polyps, genetic problems and viral infections (Allen JI, 1995; Lynch \& Lynch, 1998). Given the importance of colorectal cancer as the most common gastrointestinal cancer and the necessity of performing accurate screening, diagnosis, early treatment and reduce the economic costs, in this study tumors, polyps and certain other demographic indicators in patients were referred to Endoscopy clinic of Toos and Firoozgar in Tehran between January 2013 and May 2013 and compared with the other countries examined these variables. 


\section{Materials and methods}

In this study, informed consent was received from all patients admitted to the Endoscpy clinic of Toos and Firoozgar hospital in Tehran, Iran between January 2013 and May 2013. Fifteen patients with colorectal cancer and 20 patients with colorectal polyps were examined. Sampling was performed by endoscopic biopsy operation. Anatomic site of involvement in patients with colorectal tumor and polyp were determined during colonoscopy. The histologically type of the tumor and colorectal polyp and other features were diagnosed after pathology report. Also demographic data including age, gender, family history of cancer and etc were gathered in the form. Data was performed with SPSS software and chisquare test.

\section{Results}

From 35 patients studied, 15 patients $(42.8 \%)$ with colorectal cancer and 20 patients $(57.1 \%)$ were diagnosed with colorectal polyp. From 15 patients with colorectal cancer, 11 patients $(73.3 \%)$ male and $4(26.7 \%)$ were female, also from 20 patients with colorectal polyps, 7 patients (35\%) male and $13(65 \%)$ were female $(\mathrm{P}=0.028)$ (Table 1$)$. Statistically analysis showed a significant relationship between gender and incidence of colorectal cancer and polyps $(\mathrm{P}<$ $0.05)$. Incidence of colorectal cancer in the age group below 35 years $6.7 \%$, in the age group 35-55 years $33.3 \%$ and older than 55 years were $60 \%$, also the incidence of colorectal polyps in the age group $35-55$ years $35 \%$ and $65 \%$ were in older than 55 years $\quad(\mathrm{P}=0.503)$ (Table 1). Results showed in $80 \%$ of patients with colorectal cancer and $60 \%$ of patients with colorectal polyps body mass index (BMI) was less than $25(\mathrm{P}=0.138)$. Studies showed that the most common symptoms in patients with colorectal cancer and polyps respectively abdominal pain, weight loss, constipation and rectal bleeding. The family history of gastrointestinal cancer in patients with colorectal cancer was found in $6.7 \%$ of cases gastric cancer and $26.7 \%$ colon cancer, also in patients with colorectal polyp was found in $20 \%$ of cases gastric cancer, $15 \%$ colon and $5 \%$ pancreatic cancer $(\mathrm{P}$ $=0.305$ ). Tumor type in patients with colorectal cancer in $100 \%$ of cases are adenocarcinomas. Also check the condition of patients with colorectal cancer were identified $13.2 \%$ stage I, $19.8 \%$ stage II, 13.2\% stage III and $53.8 \%$ were stage IV disease. The type of colorectal polyp tissue in $75 \%$ hyperplastic, 15 adenomatous tubular and $10 \%$ adenomatous villuses. Anatomic site of involvement in patients 
with tumors in $33.3 \%$ of cases rectum, $20 \%$ ascending colon, $20 \%$ sigmoid, $13.3 \%$ cecum, $6.7 \%$ descending colon and $6.7 \%$ transverse colon, also anatomic site of involvement in patients with polyps in $25 \%$ of cases sigmoid, $15 \%$ rectum, $15 \%$ ascending colon, $15 \%$ cecum, $15 \%$ descending colon and $15 \%$ transverse colon (P $=0.169)$ (Table 1). Statistically analysis showed no significant association between age, body mass index (BMI), family history of gastrointestinal tract cancers and anatomic location of involvement and incidence of colorectal cancer and polyps $\quad(\mathrm{P}>0.05)$.

Table 1. Demographical features of the patients with colorectal tumor and polyp

\begin{tabular}{|l|c|c|c|}
\hline Gender & $\begin{array}{c}\text { Colorectal tumor } \\
\text { n (\%) }\end{array}$ & $\begin{array}{c}\text { Colorectal polyp } \\
\text { n (\%) }\end{array}$ & P ( $\mathbf{2})$ \\
Male & & & 0.028 \\
Female & $11(73.3 \%)$ & $7(35 \%)$ & \\
Age group & $4(26.7 \%)$ & $13(65 \%)$ & \\
Under 35 & & & 0.503 \\
$35-55$ & $1(6.7 \%)$ & $0(0 \%)$ & \\
Upper 55 & $5(33.3 \%)$ & $7(35 \%)$ & \\
Anatomic location & $9(60 \%)$ & $13(65 \%)$ & \\
Rectum & & & 0.169 \\
Ascending colon & $5(33.3 \%)$ & $3(15 \%)$ & \\
Sigmoid colon & $3(20 \%)$ & $3(15 \%)$ & \\
Cecum & $3(20 \%)$ & $5(25 \%)$ & \\
Descending colon & $2(13.3 \%)$ & $3(15 \%)$ & \\
Transvers colon & $1(6.7 \%)$ & $3(15 \%)$ & \\
Total & $1(6.7 \%)$ & $3(15 \%)$ & \\
\hline
\end{tabular}

\section{Discussion}

The findings of this study were showed that the incidence of colorectal cancer in men $(73.3 \%)$ than women $(26.7 \%)$ and the incidence of colorectal polyps in women $(65 \%)$ than men $(35 \%)$, thus colorectal cancer diseases in men and colorectal polyp diseases in women. Studies Levi and colleagues (Levi et al., 1991) also reported the incidence of colorectal cancer in men than women, which is consistent with the results of our studies. Our study showed that the incidence 
of colorectal cancer and polyps in patients aged over 55 years than other age groups studied. Results showed that $80 \%$ of patients with colorectal cancer and $60 \%$ of patients with colorectal polyps body mass index (BMI) was less than 25 . Clinical studies in patients with colorectal cancer and polyps were identified as abdominal pain, weight loss, constipation and rectal bleeding ranked first to fourth, so if this is the beginning of symptoms, rapid assessment, complete and accurate diagnosis is essential. Studies showed that $33.4 \%$ of patients with colorectal cancer and $40 \%$ of patients with colorectal polyps was found the family history of gastrointestinal cancer. Cancer screening program in patients with a family history of colorectal especially in patients with rectum and sigmoid sites cancer is essential, why hide the symptoms of this type of cancer its later shows. Studies Salttery and colleagues (Salttery et al., 1996) showed a positive family history of colorectal cancer is risk increased in aged less than 50 years. In study by Menko (Menko, 1999) family history of cancer was found in $15 \%$ of patients with tumor. Also in study by Hansen (Hansen, 1997) family history of cancer was found in $15 \%$ of patients with tumor. Mayer (Mayer, 2001) showed that the family history of cancer in $25 \%$ of patients with colorectal cancer. Weie and colleagues (Weie et al., 2004) showed positive family history is a risk factor for colorectal cancer. Results showed 100\% tumor tissue samples of adenocarcinoma and in $75 \%$ of polyp tissue samples of hyperplastic type, 15 adenomatous tubular and 10\% adenomatous villuses. Dem and colleagues (Dem et al., 2000) studied the most common type of tumor was adenocarcinoma. The present study showed that the most common anatomic site of involvement in patients with colorectal tumor was rectum $(33.3 \%)$ and in patients with colorectal polyp was sigmoid (25\%). In a study by Boyole and Lanman (Boyole \& Lanman, 2000) most common anatomic site of involvement in patients with colorectal cancer rectum. In contrast Majumdar (Majumdar, 1999) and Janaut and Kollarova (Janaut \& Kollarova, 2001) most common anatomic site involved in the discending colon of patients with colorectal tumor expressed. Currently colorectal cancer is the most common gastrointestinal cancer and one of the greatest health problems in Iran. Public education in taking seriously the warning signs of cancer, identification of the causative agents, such as cancer causing viruses and bacteria and prove their cofactor role can play an important role in improving the health status of the population.

Acknowledgements. We wish to acknowledge the very kind help of Dr. Farzad Khalili to the sample collection. This work was supported by the Endoscopy clinic of Toos and Firoozgar hospital in Tehran, Iran. 


\section{References}

[1] J.L. Allen, Molecular biology of colorectal cancer: a clinician's review, perspect Colon and Rectal Surg, 8 (1995), 181-202.

[2] P. Boyole, J. Lanman, ABC of colorectal cancer epidemiology, BMJ, 321 (2000), 805-808.

[3] R.W. Burt, Colon cancer screening, Gastroenterology, 119 (2000), 837-853.

[4] P.S. Dem, et al, Epidemiological and therapeutic aspects of rectal cancer in Senegal: 74 case at the cancer institute of daker, Dakerg Med, 45 (2000), 66-69.

[5] H. Hansen, Patients perception of symptoms in colorectal cancer, a cause of delay in diagnosis and treatment, Ugeskr Laeger, 159 (1997), 1941-1944.

[6] V. Janaut, H. Kollarova, Epidemiology of colorectal cancer, Biomed Paper, 145 (2001), 5-10.

[7] F. Levi, C. Vecchia, L. Randimbisan, V.C. Te, S. Franceschi, Patterns of large bowel cancer by subsite, age, sex and marital status, Tumori, 77 (1991), 246-251.

[8] H.T. Lynch, J.F. Lynch, Genetics of colonic cancer, Digestion, 59 (1998), 481-492.

[9] S. Majumdar, How dose colorectal cancer present? Symptom, duration and cluse of location, Am J Gast, 94 (1999), 39-45.

[10] R. Mayer, Gastrointestinal tract cancer, Harrison's principles of internal medicine, New York, 2 (2001), 581-586.

[11] F. Menko, Genetic of colorectal cancer, hereditary background of sporadic and familial colorectal cancer, Ned Tijd Schr Geneeskd, 143 (1999), 1207-1211.

[12] D.M. Parkin, F. Bary, J. Ferlay, P. Pisani, Global cancer statistics, 2002, CA Cancer J Clin, 55 (2005), 74-108.

[13] M.L. Salttery, G.D. Friedman, J.D. Potter, S. Edward, B.J. Cann, W. Sarnowitz, Adescription of age, sex and site distributions of colon carcinoma in three geograph areas, Cancer, 78 (1996), 1666-1670. 
[14] K. Weie, E. Giovannucci, K.Wu, B. Rosner, C.S. Fuchs, W.C. Willett, G.A. Colditz, Comparsion of risk factor for colon and rectal cancer, Int Cancer, 108 (2004), 433-442.

Received: March 2, 2014 